

\title{
GESTIÓN DE OBJETOS DE APRENDIZAJE A TRAVÉS DE LA RED, BASADA EN EL DESARROLLO DE COMPETENCIAS
}

Resumen: Hoy en día existen una gran cantidad de bibliotecas virtuales y repositorios de recursos en general, donde es posible acceder a diverso tipo de información, destinado a múltiples áreas de conocimiento.

Uno de esos tipos de información corresponde a los Objetos de Aprendizaje (OAs), los cuales a través de sus metadatos pueden ser localizados y recuperados según necesidades educativas específicas de los usuarios.

Los objetivos educativos están claramente orientados al desarrollo de competencias, por tanto, es importante tener en cuenta materiales que puedan potenciarlas. Debido a la caracteristica de los OAs, como unidades independientes que puedan ser reutilizadas, se propone un modelo de diseño que oriente la creación de estos recursos según niveles de conocimiento necesarios para el desarrollo de competencias.

Para promover la búsqueda de estos recursos en base a niveles de conocimiento, se presenta una propuesta para su catalogación a través de metadatos, que considere además de los niveles de conocimiento, su clasificación por competencias.

Palabras clave: gestión del conocimiento; objetos de aprendizaje; aprendizaje basado en competencias; metadatos.

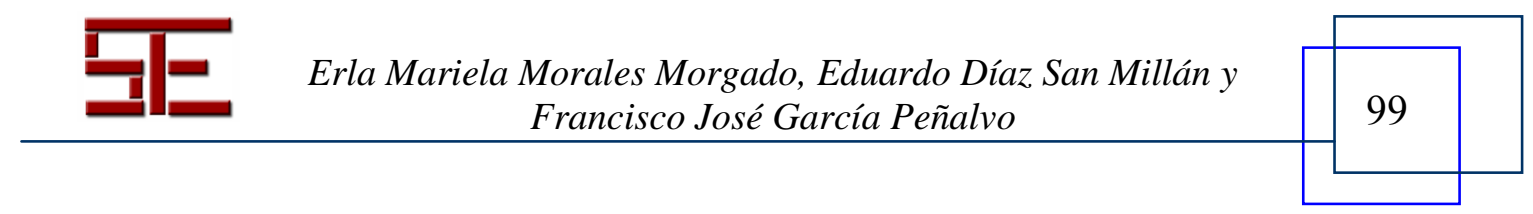




\section{ONLINE LEARNING OBJECTS MANAGEMENT BASED ON COMPETENCIES DEVELOPMENT}

Abstract: Nowadays there are a large amount of virtual libraries and repositories of resources, where people can access an great variety of information in multiple fields of knowledge.

One of these types of data is Learning Objects (LOs), which contain metadata to locate and recover them, according to the specific educational needs of users.

Educational objectives are clearly oriented to skills development. Therefore, it is important to consider educational materials that promote their use. Due to the LOs characteristics as independent units that can be reused, we propose a guide to LOs design with the aim of developing these resources according to levels of knowledge required for competency development.

In order to promote the learning objects management based on skill levels, we suggest that their metadata be cataloged by level of knowledge and competencies classification.

Keyword: knowledge management, learning objects, competence based learning, metadata.

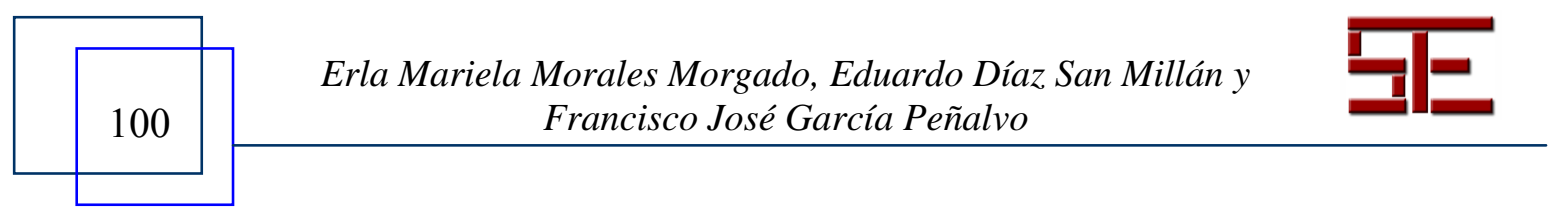




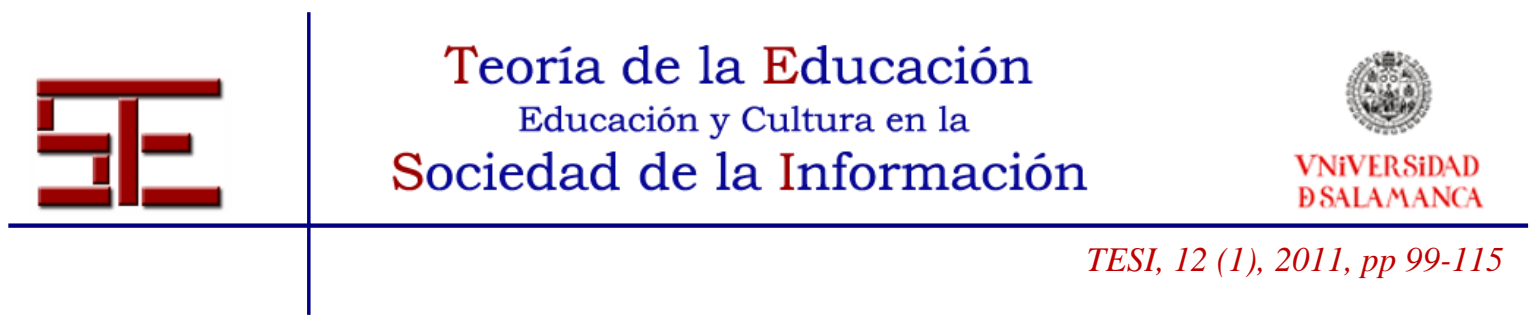

\section{GESTIÓN DE OBJETOS DE APRENDIZAJE A TRAVÉS DE LA RED, BASADA EN EL DESARROLLO DE COMPETENCIAS}

Fecha de recepción: 03/12/2010; fecha de aceptación: 16/02/2011; fecha de publicación: 31/03/2011

Erla Mariela Morales Morgado

erlamorales@usal.es

Universidad de Salamanca

Eduardo Díaz San Millán

eduardodiaz@usal.es

Universidad de Salamanca

Francisco José García Peñalvo

fgarcia@usal.es

Universidad de Salamanca

\section{1.- GESTIÓN DE RECURSOS EDUCATIVOS}

La gestión del conocimiento es, hoy en día, un aspecto clave para buscar, procesar y recuperar información adecuada según las necesidades de los usuarios. La Red es una de las principales fuentes de información para la formación, por tanto, se requiere de una adecuada gestión de los recursos educativos para promover aprendizajes de calidad, que permitan a los estudiantes desenvolverse de forma activa y eficiente en esta era de la información.

La posibilidad que ofrecen los estándares educativos de gestionar los Objetos de Aprendizaje (OAs), facilitando su interoperabilidad y reutilización en diversas plataformas, y la existencia de lenguajes de modelado educativo que permiten además estructurarla de manera que tenga sentido pedagógico, abren una importante posibilidad de mejora para los sistemas de educación en línea (Morales, 2010).

Para conseguir una adecuada gestión de los OAs, es importante catalogarlos de forma correcta a través de metadatos. El estándar de metadatos de referencia para el habla hispana es LOM-ES, una adaptación del estándar LOM al idioma Español, el cual cuenta también con la categoría "uso educativo", que agrupa las características educativas y pedagógicas de los objetos, como, por ejemplo: destinatarios, dificultad, nivel de interactividad, etc.

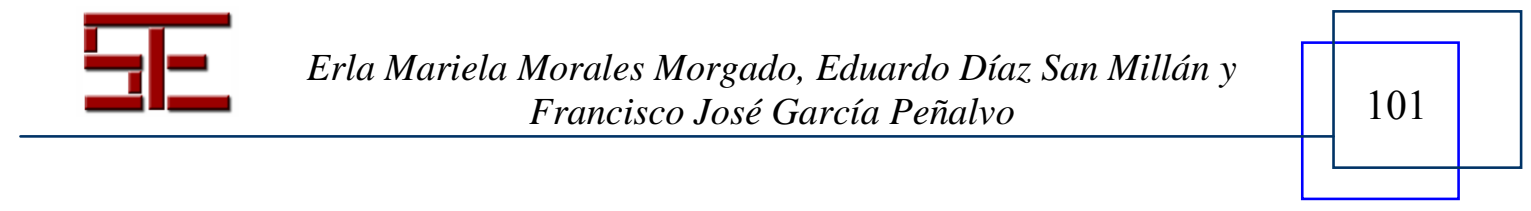




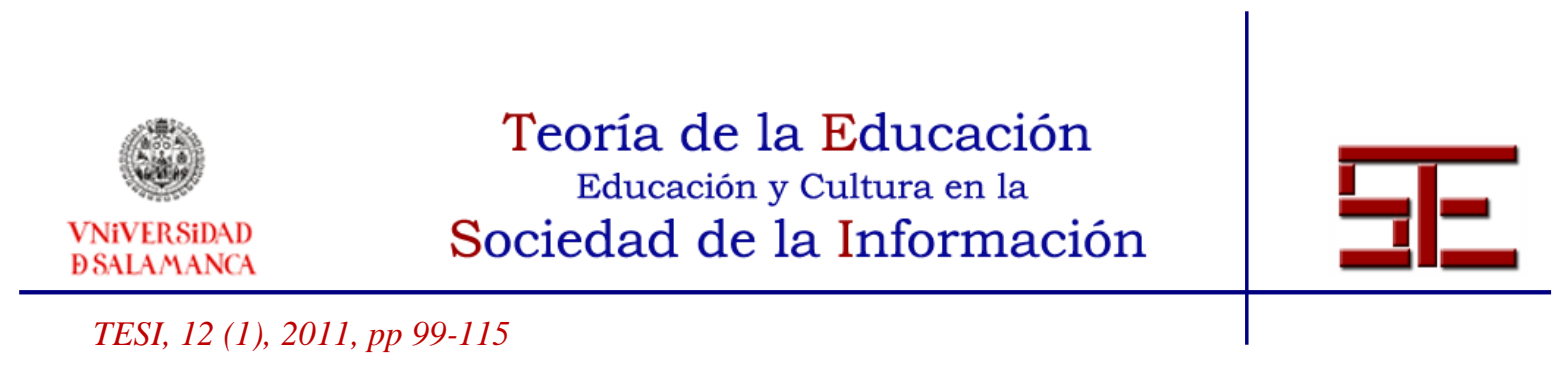

La catalogación de los recursos viene determinada en algunos casos por los repositorios de almacenamiento donde estos se encuentran almacenados, tal como se explicará en el siguiente apartado.

\section{1.- Repositorios de almacenamiento de recursos educativos}

Hoy en día, existen una gran cantidad de bibliotecas virtuales y repositorios de recursos en general, donde es posible acceder a diverso tipo de información, destinado a múltiples áreas de conocimiento.

Los Objetos de Aprendizaje son agrupados y almacenados en Repositorios de Objetos de Aprendizaje (ROA). Formalmente, un ROA es una colección de OAs, que tienen información detallada de sí mismos (metadatos) accesible vía Internet (López \& García, 2005). De éstos, se identifican dos tipos (Downes, 2002). Por una parte, los que contienen OAs y sus metadatos, en donde los objetos y sus descriptores se encuentran dentro de un mismo sistema e incluso dentro de un mismo servidor. Por otra parte, los que contienen sólo los metadatos, en este caso el repositorio contiene sólo los descriptores y se accede al OA a través de una referencia a su ubicación física, que se encuentra en otro sistema o repositorio de OAs.

La importancia de gestionar los recursos para atender necesidades específicas de los usuarios ha sido bien considerada en el ámbito educativo. Un ejemplo concreto es el proyecto Agrega (http://www.proyectoagrega.es), el cual alberga un repositorio de contenidos, que almacena contenidos empaquetados conforme a una extensión del esquema SCORM 2004 (ADL, 2005), el cual permite referencias a recursos externos y con catalogación basada en el perfil de aplicación LOM-ES (http://www.lom-es.es/).

El proyecto Agrega tiene entre sus principales objetivos definir una referencia estándar de catalogación, empaquetado y publicación de contenidos educativos digitales. En cuanto a la catalogación de los recursos, Agrega cuenta con un catalogador básico, que permite a los docentes añadir fácilmente y de forma concisa los siguientes datos: título, idioma, descripción, tipo de recurso e idioma del destinatario.

Otro importante aspecto que destacar en Agrega es que añade la inserción curricular, como mecanismo de datación del objeto según su inserción en el sistema curricular educativo español. Es un subconjunto del estándar de metadatos LOM-ES. Su

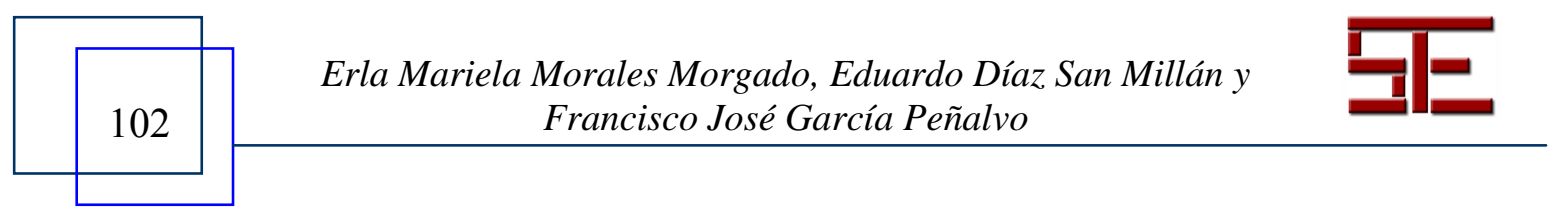




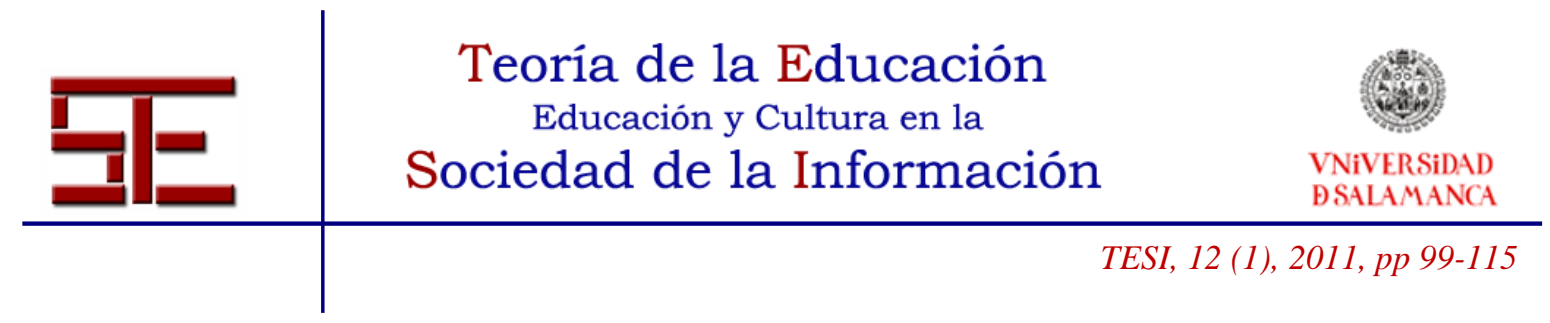

utilización está pensada para usuarios que no tengan conocimiento de los estándares LOM-ES y SCORM.

Otros repositorios de OAs que podemos encontrar son:

- IIEP-UNESCO Wiki of OER repositories: UNESCO/IIEP hospeda un Wiki que ofrece una lista de varios portales, puertas de acceso y repositorios. Ofrece un listado de enlaces a iniciativas OER, recursos y herramientas. Se compiló siguiendo el primer foro de debate IIEP sobre Open Educational Resources (24 de octubre - 2 de diciembre 2005). Proporciona acceso a una selección de aproximadamente 30 repositorios de objetos de aprendizaje abierto, la mayoría de nivel universitario.

- MERLOT (Multimedia Educational Resource for Learning and Online Teaching), repositorio centralizado que contiene solo metadatos. Es independiente y funciona como un portal de objetos de aprendizaje. Evalúa los objetos mediante evaluación por pares. Gratis y abierto. http://www.merlot.org/.

- CAREO (Campus Alberta Repository of Educational Objects), repositorio centralizado de objetos de aprendizaje multidisciplinarios de profesores de Alberta (Canadá). Es independiente que da acceso a objetos remotos y locales a través de los metadatos contenidos en su colección. Gratis y abierto. http://www.careo.org/.

- CLOE (Co-operative Learning Object Exchange), modelo cooperativo para el desarrollo, uso y reutilización de objetos de aprendizaje. http://cloe.on.ca/

- SMETE (Science, Mathematics, Engineering and Technology Education), repositorio distribuido, que integra varias bibliotecas de recursos educativos. Acceso es libre para la consulta. http://www.smete.org/smete/.

- GEM (Gateway to Educational Materials) es un proyecto del Departamento de Educación de los EEUU. Orientada a la interoperabilidad entre múltiples bases de datos a través del uso de módulos que extraen los metadatos de los objetos en su formato GEM. http://www.thegateway.org/.

- POOL (Portals for Online Objects in Learning), consorcio de organizaciones educativas para crear un gran repositorio distribuido. http://www.edusplash.net/.

- CeLeBraTe (Context eLearning with Broadband Technologies), Plantea un repositorio centralizado con una administración parcial de los metadatos por el usuario. http://celebrate.eun.org/eun.org2/eun/en/index_celebrate.cfm/.

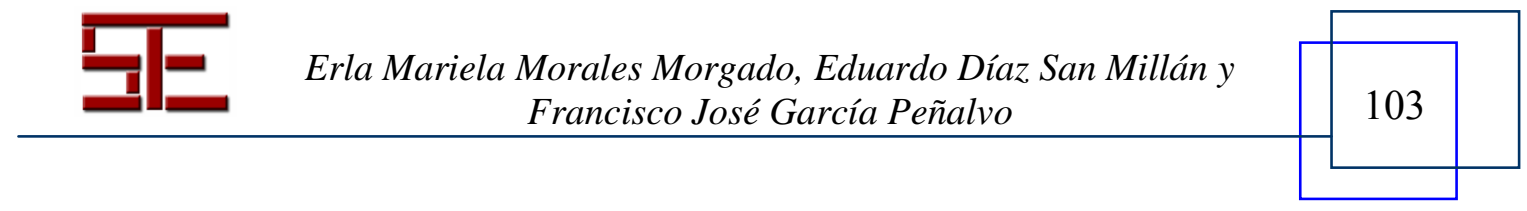






- ELENA/Edutella, parte de un proyecto mayor en el que ELENA es una capa de la infraestructura en la que se conectan aplicaciones con tipos diferentes de repositorios, modelos de búsqueda y diferentes esquemas de metadatos. http://www.elena-project.org/.

- EduSourceCanada, este proyecto es una propuesta para crear una red de ROA en Canadá, uniendo los principales repositorios creados en este país. http://www.edusource.cal.

- Biblioteca Escolar Digital. Repositorio gratuito de la Fundación Germán Sánchez Ruipérez. Se pueden obtener OA de educación primaria, secundaria o bachillerato. http://www.bibliotecaescolardigital.es/.

\section{2.- DISEÑO DE OBJETOS DE APRENDIZAJE EN BASE A COMPETENCIAS}

Como bien es sabido, uno de los principales objetivos del plan Bolonia es aumentar la competitividad de la universidad europea. Para lograr este objetivo, se ha propuesto involucrar a los estudiantes en una experiencia educativa, basada en el desarrollo de competencias, que les ayude a alcanzar la excelencia, tanto en el saber (conocimientos), saber hacer (competencias) y saber ser (actitudes), con la finalidad de alcanzar un alto rendimiento en su desempeño profesional.

Una competencia es definida como "una característica subyacente en una persona que está causalmente relacionada con el desempeño, referido a un criterio superior o efectivo, en un trabajo o situación" (De Miguel, 2006; Spencer \& Spencer, 1993), (Villa $\&$ Poblete, 2008). Entre los componentes de una competencia se encuentran:

- Conocimientos: Adquisición sistemática de conocimientos, clasificaciones, teoría, etc. Relacionados con materias científicas o área profesional.

- Habilidades y destrezas: Entrenamiento en procedimientos metodológicos aplicados relacionados con materias científicas o área profesional (organizar, aplicar, manipular, diseñar, etc.).

- Actitudes y valores: Actitudes y valores necesarios para el ejercicio profesional: responsabilidad, autonomía, iniciativa ante situaciones complejas, coordinación, etc.

Debido a lo anteriomente expuesto, el diseño de materiales educativos orientados al desarrollo de competencias cobra hoy en día una especial importancia. El diseño de OAs es un tema que no está claramente definido y, debido a su característica de unidades independientes, sería interesante contar con una propuesta que ayude a un






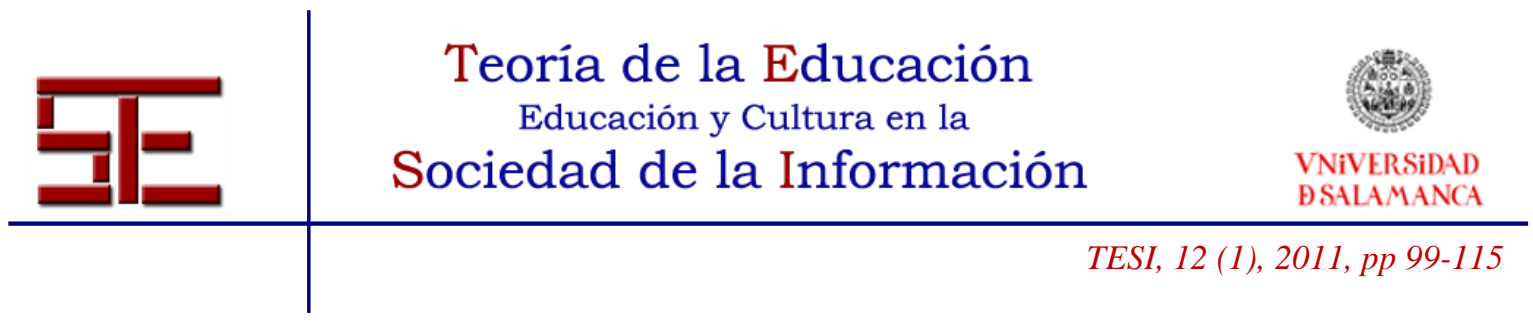

diseño, que permita reutilizarlos como unidades autocontenidas, asociadas a los diversos tipos de conocimientos que se requiere para el desarrollo de competencias.

\section{1.- Diseño de unidades independientes para el desarrollo de competencias}

Debido a la diversidad de definiciones de OAs, no hay un modelo de diseño adoptado por excelencia, sin embargo, para que éstos logren una mayor reusabilidad es conveniente que su diseño y creación se ajuste a unidades mínimas de aprendizaje. Si se dotan además de niveles de contenidos determinados, como, por ejemplo, de tipo "conceptual" o "procedimiental", podremos facilitar la busqueda y recuperacion de recursos más adecuados a las necesidades específicas de aprendizaje.

Con el objetivo de orientar el diseño de OAs hacia el desarrollo de competencias, se presenta a continuación una propuesta de diseño para una lección específica, orientada al desarrollo de contenidos, básicos para el desarrollo de competencias, los cuales pueden conformar en su conjunto unidades mayores de aprendizaje.

Estos grupos de OAs que darán forma a nuevas unidades educativas de diversos niveles, deben ser clasificados para saber concretamente qué tipo de OA se está gestionando.

Considerando el nivel de granularidad o tamaño propuesto por IEEE LOM (2002), en esta propuesta se sugiere la siguiente clasificación (Morales, García \& Barrón, 2007).

- OA nivel 1: Se refiere al nivel más atómico o granular de agregación, ej: imágenes, segmentos de texto o vídeos (IEEE LOM, 2002).

- OA nivel 2: Una lección con un objetivo de aprendizaje específico, con un tipo de contenido (datos y conceptos, o procedimientos y procesos, o reflexión y actitud) y finalmente y actividades de evaluación y práctica (opcional).

- OA nivel 3: Un módulo de aprendizaje compuesto por un conjunto de lecciones (OAs de nivel 2).

- OA nivel 4: Un curso compuesto por un conjunto de módulos (OAs de nivel 3)

Para diseñar una lección en un $\mathrm{OA}$, consideramos el tamaño que engloba los requisitos mínimos necesarios para que pueda ser reutilizado como unidad educativa, es decir, el nivel 2. Para que el diseño tenga sentido pedagógico, sugerimos los siguientes componentes, representados de forma gráfica a través de la Figura 1.

- Introducción al tema, en donde se deben definir los objetivos según el nivel cognitivo y el nivel de dificultad. El temario estará asociado a tipo de contenido

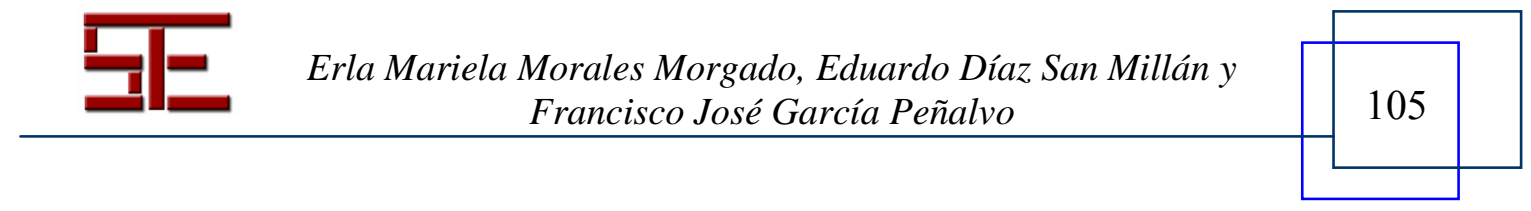






específico. La indicación explícita de las palabras claves relacionadas al OA, puede ser de utilidad para buscar otros recursos relacionados.

- Contenidos relacionados a un tipo específico de conocimiento, necesarios para lograr una determinada competencia, esto es:

○ "saber qué" (datos y conceptos) o

○ "saber cómo" (procedimientos y procesos) o

○ "saber acerca de" (o reflexión y actitud).

- Actividades de práctica y evaluación, que pueden ser opcionales según sea el caso, en ellas se especifican claramente tipos de actividades, modalidad de trabajo y estrategias.

Teniendo en cuenta el diseño indicado anteriormente, es posible componer unidades mayores de aprendizaje, como, por ejemplo, una unidad didáctica y un curso.

Para diseñar una unidad didáctica o módulo, se tendrá en cuenta el nivel de granularidad número 3, definido en la sección anterior, dirigido en este caso a desarrollar una o varias competencias.

Para promover este objetivo, debería estar compuesta por varias lecciones, las cuales requieren contener, como mínimo y de forma independiente, los tres tipos de contenidos mencionados, que se necesitan para lograr una determinada competencia.

Cuando se construye una unidad didáctica o curso, basada en la reutilización de unidades más pequeñas, es importante considerar una manera de conectarlos como un todo, orientado a la consecución de mayores objetivos, como, por ejemplo:

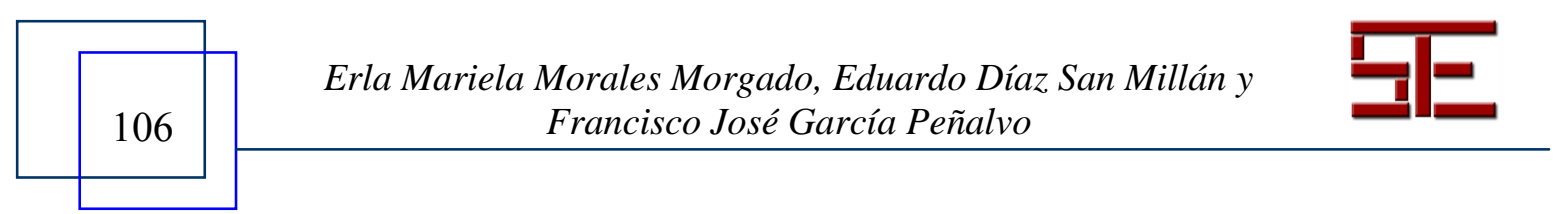



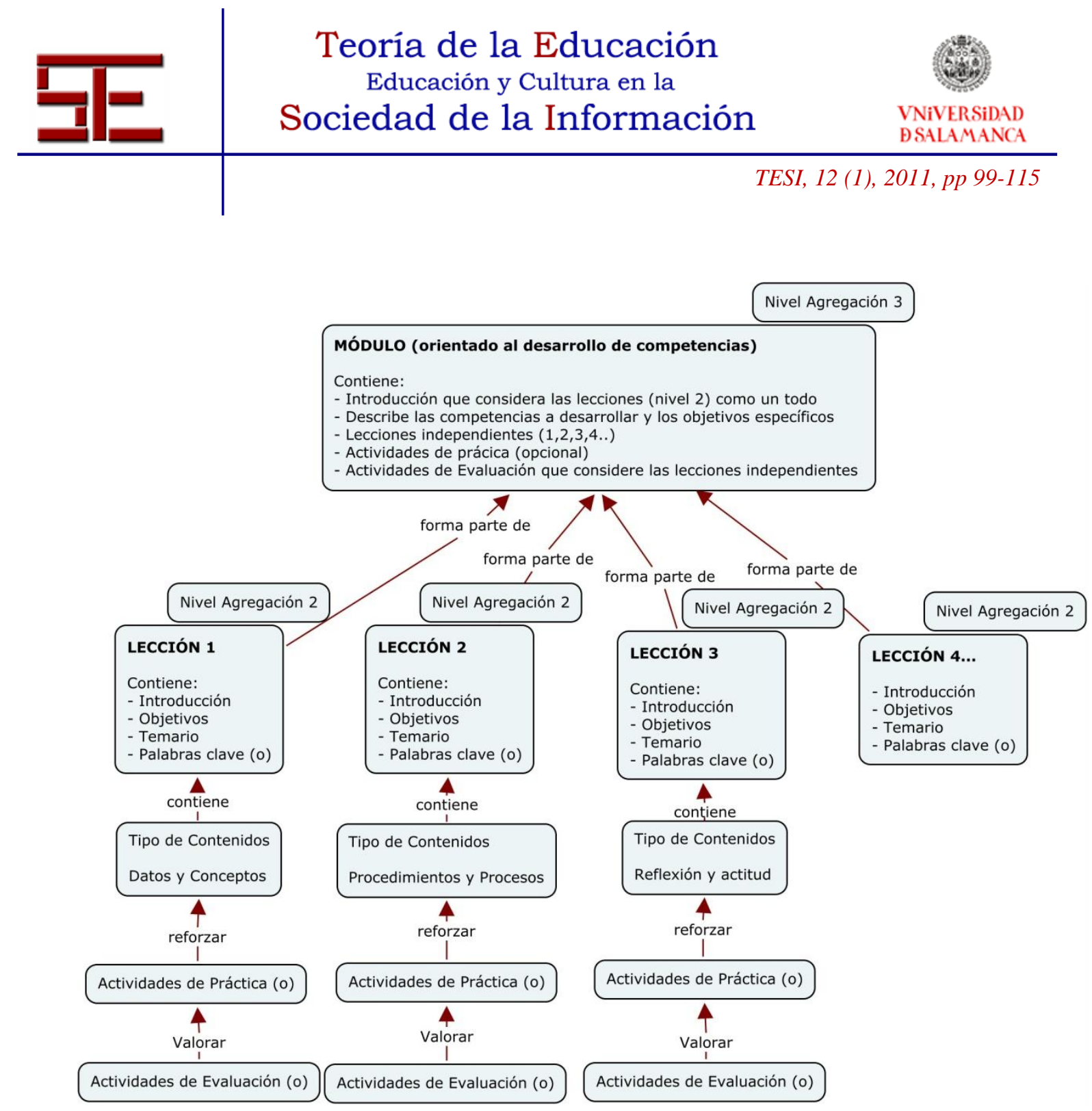

Figura 1. Propuesta de diseño de un módulo orientado al desarrollo de competencias.

- Introducción a la unidad didáctica, indicando las competencias que se pretenden desarrollar, las lecciones que lo componen, objetivos necesarios para lograr un determinado nivel cognitivo, el nivel de dificultad, etc.

- Actividades de práctica y evaluación (según sea el caso), que ayuden a reforzar los conocimientos de cada nivel de conocimiento y conectar los tres 




en conjunto para promover el logro de las competencias descritas en la unidad didáctica.

Sobre la base de lo anteriormente expuesto, el diseño de un curso podría estar compuesto por unidades didácticas independientes, orientadas al desarrollo de diversas competencias y éstas, a su vez, compuestas por lecciones autocontenidas, que puedan ser reutilizadas según las necesidades educativas.

\section{2.- Propuesta de catalogación de unidades independientes según un nivel de conocimiento específico}

Dentro de la categoría de metadatos LOM-ES, no hay un elemento específico que se refiera al tipo de contenido de los OAs. Si bien es cierto, hay otros elementos que permiten introducir texto libre, como, por ejemplo, la descripción del recurso, pero esta opción se utiliza más para comentar las condiciones de utilización de los recursos educativos.

El estándar LOM-ES tiene una categoría llamada "9.Clasificacion", que permite definir clasificaciones específicas para los recursos, según determinados propósitos. Ante la importancia de establecer los diversos niveles de conocimiento, es interesante incluir este propósito como posibilidad de clasificación de los OAs.

Sobre esta base, se presenta una propuesta para la clasificacion de los OAs con el propósito "Tipo de Contenido", basado en los niveles de conocimiento mencionados para el desarrollo de competencias.

La propuesta se presenta en las Tablas 1, 2 y 3, las cuales se basan en los elementos o campos de la categoría "9.Clasificación" de LOM-ES. En cada una de ellas, se propone una definición de los datos que pueden ser agregados para clasificar los niveles de conocimiento mencionados.

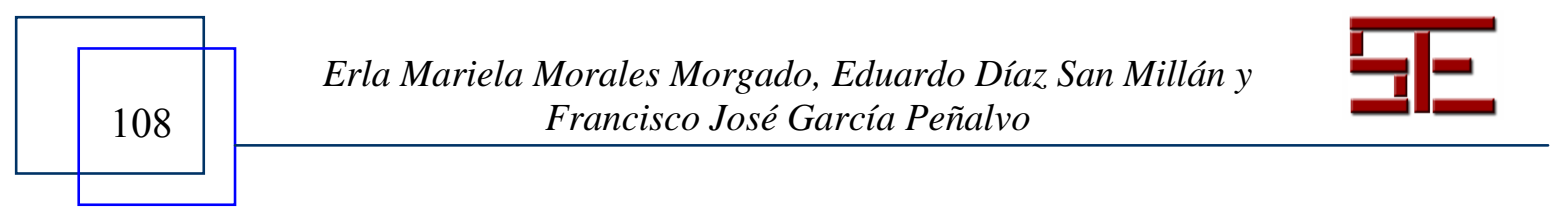




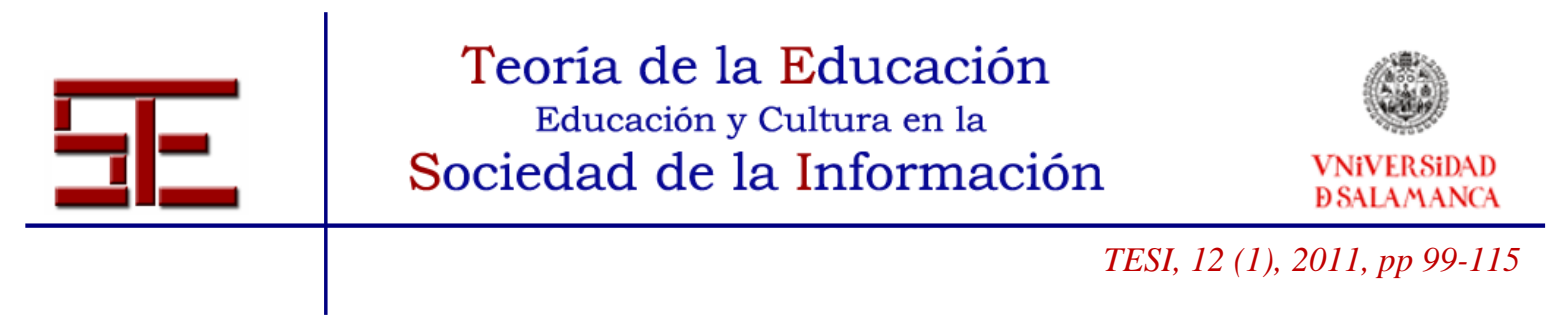

\begin{tabular}{|l|l|l|}
\hline \multicolumn{1}{|c|}{$\mathbf{N}$} & \multicolumn{1}{|c|}{ Nombre } & \multicolumn{1}{|c|}{ Ejemplo } \\
\hline 9 & Clasificación & \multicolumn{1}{|c|}{} \\
\hline $\mathbf{9 . 1}$ & Propósito & Tipo de Contenido \\
\hline 9.2 & $\begin{array}{l}\text { Ruta } \\
\text { Taxonómica }\end{array}$ & \\
\hline 9.2 .1 & Fuente & TP_DC=datos y conceptos \\
\hline 9.2 .2 & Taxón & TP_DC \\
\hline 9.2 .2 .1 & Identificador & Datos y Conceptos \\
\hline 9.2 .2 .2 & Entrada & $\begin{array}{l}\text { Datos: grupo de objetos, símbolos, ideas o } \\
\text { eventos que son definidos por una simple } \\
\text { palabra o término } \\
\text { Conceptos: Principios abstractos, métodos } \\
\text { generales, teoremas, etc. }\end{array}$ \\
\hline 9.3 & Descripción \\
\hline 9.4 & Palabras clave & $\begin{array}{l}\text { Nombres, datos, fechas, hechos, características, } \\
\text { principios abstractos, métodos generales, } \\
\text { teoremas }\end{array}$ \\
\hline
\end{tabular}

Tabla 1. Propuesta para clasificar los OAs según el tipo de contenidos "datos y conceptos", conforme a la categoría "9.Clasificación" de LOM-ES.

La Tabla 1 muestra los elementos de la categoría de metadatos "9. Clasificación” de LOM-ES, adaptado a una propuesta de clasificación, según el tipo de contenidos "datos y conceptos". A través del elemento "9.2.1 Fuente" se define el nombre del sistema de clasificación, que en este caso se ha definido como "Tipo Contenido".

Un taxón es un término concreto dentro de una taxonomía. El taxón puede ser de tipo alfanumérico para ser utilizado como referencia estandarizada y así evitar equivocaciones al momento de buscar los recursos bajo este criterio. Sobre esta base, se ha definido, a modo de ejemplo, en el elemento "9.2.2 Taxón" el siguiente valor: "TC_DC es Datos y Conceptos", en donde TC corresponde a Tipo de Contenido y DC a Datos y Conceptos.

El elemento "9.2.2.1 Identificador" corresponde al identificador del taxón, tal como un número o una combinación de letras proporcionadas por la fuente de la taxonomía, en

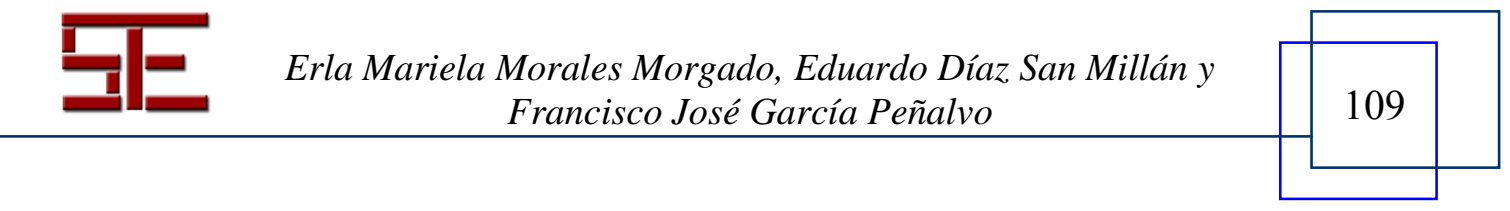






este caso, se ha definido, a modo de ejemplo, como: Tipo Contenido_Datos y Conceptos $=\mathrm{TC} \mathrm{DC}$.

El elemento "9.3 Descripción", explica la clasificación definida, es así como se ha descrito qué se entiende por "datos y conceptos", al que hace referencia el OA, de esta manera es posible conocer mejor el tipo de información al que hace referencia el OA.

Es importante destacar que estas definiciones presentadas constituyen sólo una referencia para describir el tipo de contenido. Los docentes pueden dar su propia descripción acerca de cada uno de ellos.

Finalmente, a través del elemento "9.4 Palabras clave", se definen algunos ejemplos de términos conceptuales, que pueden ser utilizados para su búsqueda según esta clasificación.

Siguiendo esta misma línea, las Tablas 2 y 3 presentan un ejemplo sobre la clasificación de los OAs para los tipos de contenidos "Procedimientos y Procesos" y "reflexión y actitud" respectivamente.

\begin{tabular}{|l|l|l|}
\hline \multicolumn{1}{|c|}{$\mathbf{N}^{\mathbf{0}}$} & \multicolumn{1}{c|}{ Nombre } & \multicolumn{1}{c|}{ Ejemplo } \\
\hline 9 & Clasificación & \multicolumn{1}{c|}{} \\
\hline $\mathbf{9 . 1}$ & Propósito & Tipo de Contenido \\
\hline 9.2 & Ruta Taxonómica & \\
\hline 9.2 .1 & Fuente & TC_PP = procedimientos y procesos* \\
\hline 9.2 .2 & Taxón & TC_PP \\
\hline 9.2 .2 .1 & Identificador & Procedimientos y Procesos \\
\hline 9.2 .2 .2 & Entrada & $\begin{array}{l}\text { Procedimientos: Pasos secuenciados que } \\
\text { describen una tarea, etc. } \\
\text { Procesos: Fases secuenciadas que describen } \\
\text { cómo funciona un sistema }\end{array}$ \\
\hline 9.3 & Descripción & Procesos, procedimientos, pasos \\
\hline 9.4 & Palabras clave &
\end{tabular}

Tabla 2. Propuesta para clasificar los OAs según el tipo de contenidos "Procedimientos y Procesos", conforme a la categoría "9.Clasificación" de LOM-ES.

- en donde TC_PP corresponde a : Tipo de Contenido Procedimientos y Procesos






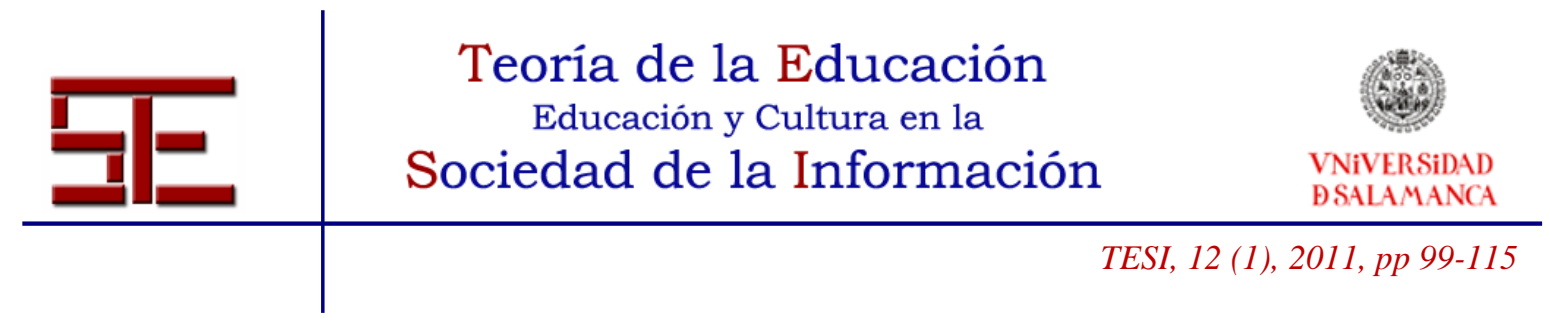

Los contenidos descritos en el elemento "9.3. Descripción" presentan de forma resumida la diferencia entre procedimientos y procesos, pero ambos, en mayor o menor medida, están relacionados con el saber hacer.

Las palabras claves son sólo un ejemplo de una forma de identificar la búsqueda de OAs, basados en este tipo de nivel de conocimiento.

\begin{tabular}{|l|l|l|}
\hline \multicolumn{1}{|c|}{$\mathbf{N}$} & \multicolumn{1}{|c|}{ Nombre } & \multicolumn{1}{c|}{ Ejemplo } \\
\hline 9 & Clasificación & \multicolumn{1}{|c|}{} \\
\hline $\mathbf{9 . 1}$ & Propósito & Tipo de Contenido \\
\hline 9.2 & Ruta Taxonómica & \\
\hline 9.2 .1 & Fuente & TC_RA03 = reflexión y actitud ${ }^{*}$ \\
\hline 9.2 .2 & Taxón & TC_RA03 \\
\hline 9.2 .2 .1 & Identificador & Reflexión y Actitud \\
\hline 9.2 .2 .2 & Entrada & $\begin{array}{l}\text { Promueven el desarrollo de actitudes, } \\
\text { valores y normas, por tanto, el material a } \\
\text { seleccionar debe inducir a la reflexión y la } \\
\text { crítica }\end{array}$ \\
\hline 9.3 & Pescripción & $\begin{array}{l}\text { Reflexión, actitud, toma de decisiones, } \\
\text { valores, normas }\end{array}$ \\
\hline
\end{tabular}

Tabla 3. Propuesta para clasificar los OAs según el tipo de contenidos "Reflexión y Actitud", conforme a la categoría "9.Clasificación" de LOM-ES.

- en donde: TC_RA corresponde al Tipo de Contenido de Reflexión y Actitud

La recuperacion de OAs desde un repositorio en donde se indique el tipo de contenido al que corresponde ese OA constituye además una valiosa información, para que los docentes puedan decidir si el recurso recuperado es adecuado o no para reutilizarlo junto a otros OAs y construir nuevas unidades didácticas y cursos.

Además de la clasificación descrita anteriormente, los OAs pueden ser catalogados por competencias, en este caso, la búsqueda de recursos arrojaría unidades didácticas completas, que contengan los tres niveles de conocimiento descritos anteriomente. A continuación, se presenta una propuesta de catalogación y búsqueda.

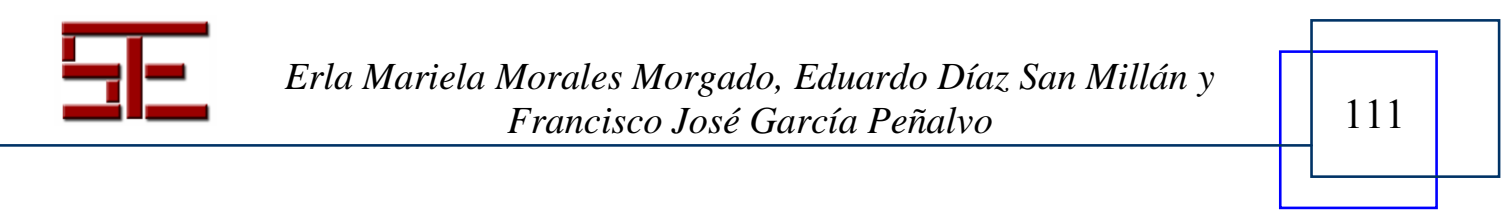




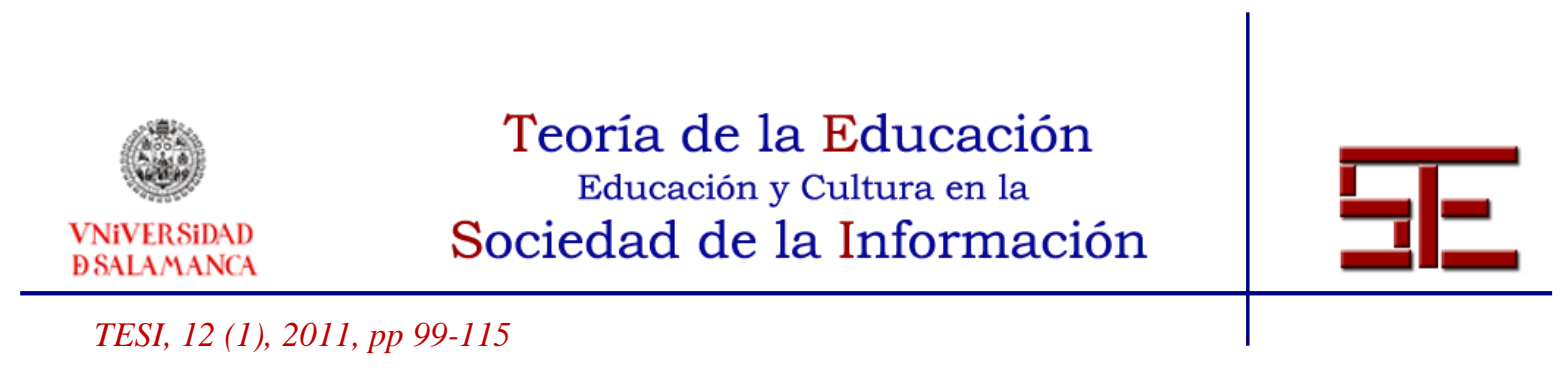

\section{3.- Propuesta de catalogación y búsqueda en base a competencias}

El aprendizaje en base a competencias es hoy en día una necesidad para cualquier tipo de organización. Es así como existen en la actualidad diversas iniciativas que promueven la estandarización de competencias, como el caso del proyecto tuning (http://tuning.unideusto.org/tuningal/), coordinado por diversas Universidades latinoamericanas y europeas, que tiene como uno de sus principales objetivos desarrollar pefiles profesionales, en términos de competencias genéricas y relativas a cada área de estudios, incluyendo destrezas, conocimientos y contenido en las áreas temáticas que incluye el proyecto.

Otro caso concreto es el catálogo nacional de cualificaciones profesionales (CNCP) (http://www.educacion.es/educa/incual/ice_catalogoWeb.html), el cual comprende las cualificaciones más significativas del sistema productivo español, organizadas en familias y niveles profesionales.

Sobre la base de determinadas competencias estandarizadas, como las que promueve el proyecto tuning y el catálogo (CNCP), es posible clasificar OAs a través de la categoría de metadatos "9.Clasificación", tal como el ejemplo descrito en el apartado anterior. Debido a la organización jerárquica que presentan las competencias estandarizadas, su clasificación a través de los metadatos puede considerar las competencias generales y de forma secundaria y dependiente, las competencias de las áreas de conocimiento específicas.

La catalogación por competencias puede ser complementada además por un conjunto de palabras clave, a través de la categoría General de LOM-ES. Para relacionar la búsqueda entre las palabras clave y competencias, es necesario desarrollar un panel mediante una rúbrica de asociación palabra y competencia junto a su descripción.

\section{3.- CONCLUSIONES}

Las posibilidades que nos plantean las tecnologías hoy en día para gestionar la información son innumerables, pero pocas veces se aprovecha adecuadamente su potencial para solucionar prolemas reales que aquejan desde hace mucho tiempo a las organizaciones para ofrecer a sus empleados la información adecuada para su formación.

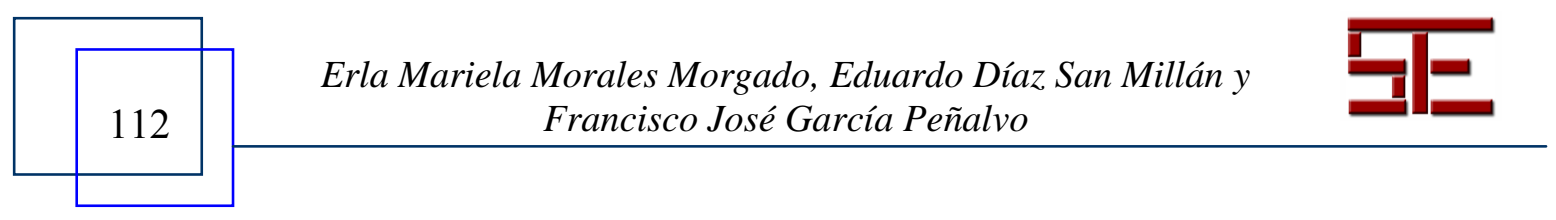




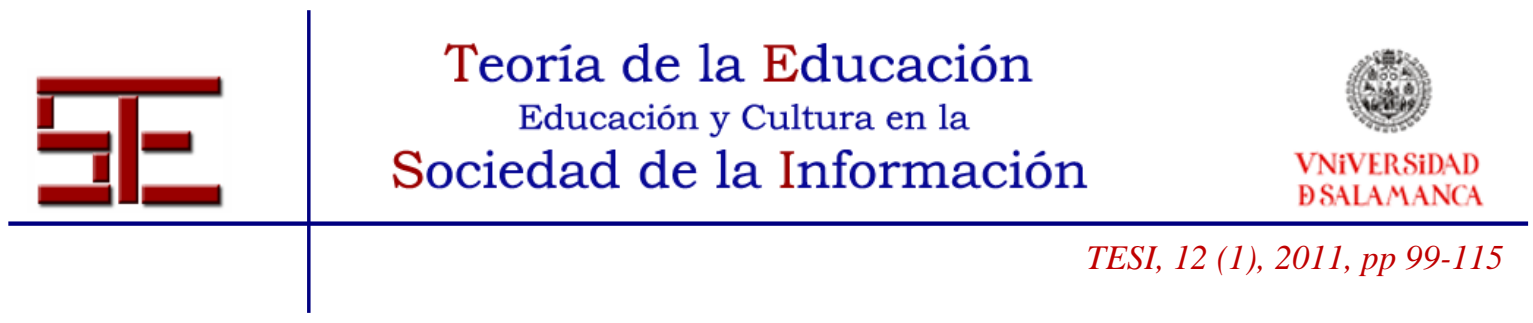

Los Objetos de Aprendizaje constituyen una alternativa interesante para gestionar materiales educativos, en donde los docentes pueden crear y compartir sus recursos, además de buscar y recuperar los que han sido creados por otros.

La propuesta descrita en este artículo para la clasificación de los OAs según determinados niveles de conocimiento y competencias constituye una interesante alternativa, incluso como una mejora para el sistema de catalogación del proyecto Agrega. Es así como se podría agregar una nueva categoría al árbol curricular del proyecto, dos nuevas fuentes de clasificación y búsqueda. La primera, basada en un sistema de competencias, establecido según adaptación a las competencias generales definidas por el proyecto Tuning y la segunda como una categoría secundaria que dependa de la anterior, basada en los sistemas de competencias de las áreas de conocimiento específicas.

También es posible utilizar un catalogador avanzado, basado en el estándar de metadatos LOM-ES, en donde las palabras clave pueden ser definidas a través de "folksonomías controladas", es decir, etiquetas colectivas agregadas por los usuarios, tal como se realiza en diversos sistemas de clasificación social, como, por ejemplo: enlaces favoritos de páginas web (del.icio.us), fotografías (flickr), etc.

La estructura de palabras clave (tags) relacionadas con las competencias, estaría asociada a la categoría "general" y a "palabra clave". Este campo se propone utilizarlo a pesar de que no sea necesario en LOM-ES. Y se espera que conviva sin inconvenientes con las palabras clave que describen el tema principal del objeto educativo, permitiendo realizar búsquedas mediante tags relacionados con la temática del objeto y con las competencias buscadas.

Siguiendo con el caso de Agrega, la clasificacion por competencias sugerida permitiría, por una parte, mejorar la localización y la adaptación de los objetos del repositorio en el ámbito preuniversitario, proponiendo objetos que han de facilitar la adquisición de competencias clave en los posteriores estudios de grado. Por otra parte, permitiría mejorar, también, la localización y la adaptación de los objetos del repositorio a las competencias demandadas en el ámbito empresarial, formando personas más adaptadas a los requisitos de los procesos de empleabilidad.

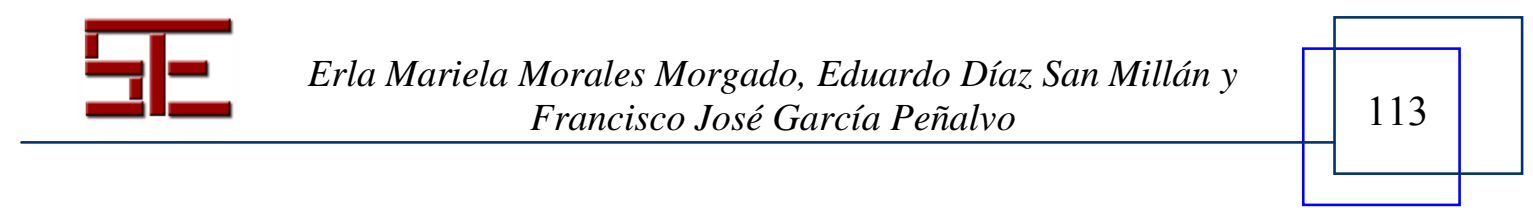




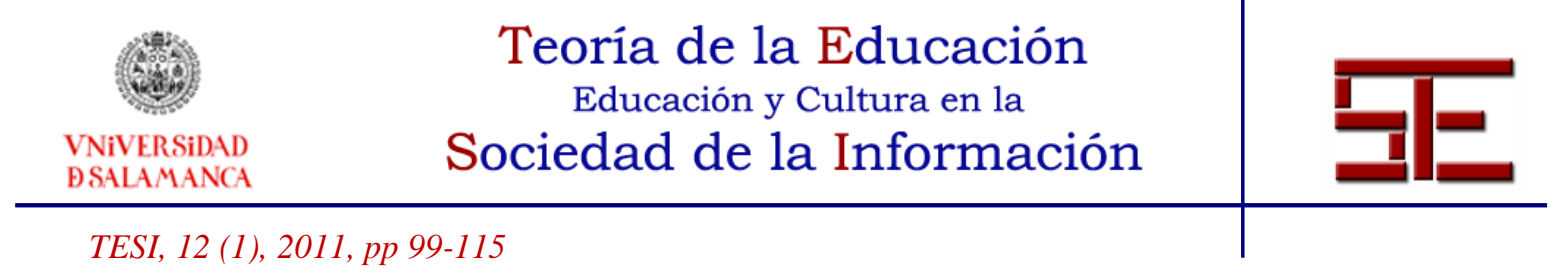

Finalmente, la propuesta podría facilitar al docente una mecánica de etiquetación sencilla y que, en buena medida, evite la incertidumbre al disponer de plantillas de ayuda.

\section{Agradecimientos}

Este trabajo está subvencionado por el Ministerio de Industria, Turismo y Comercio (proyecto TSI-020302-2009-35), por el Ministerio de Ciencia e Innovación (proyecto TIN2010-21695-C02-01) y por la Junta de Castilla y León a través del proyecto de excelencia GR47.

\section{4.- BIBLIOGRAFÍA}

ADL (2005). Advanced Distributed Learning Initiative. Disponible en http://www.adlnet.org.

De Miguel Díaz, M. (2006). Modalidades de enseñanza centradas en el desarrollo de competencias. Modalidades de enseñanza para promover el cambio metodológico en el Espacio Europeo de Educación Superior. Ediciones Universidad de Oviedo.

Downes, S. (2002). Design and Reusability of Learning Objects in an Academic Context: A New Economy of Education?. Moncton, Canada: National Research Council Extraído el 8 de agosto de 2004, de http://www.downes.ca/files/milan.doc.

IEEE LOM (2002). IEEE 1484.12.1-2002 Standard for Learning Object Metadata. Extraído en June, 2007, from http://ltsc.ieee.org/wg12.

López, G. \& García, F. J. (2005). Repositorios de Objetos de Aprendizaje basados en Estándares. En Polo Márquez, A y Herrera Viedma, E. (Eds.), Actas de las V Jornadas de Bibliotecas Digitales, JBiDi'2005 (Granada, 13-16 de septiembre de 2005). (pp. 9398). Thomson.

Morales, E. M. (2010). Gestión del Conocimiento en Sistemas e-learning, Basado en Objetos de Aprendizaje Cualitativa y Pedagógicamente definidos. Colección Vítor, 273. Ediciones Universidad de Salamanca y Erla Mariela Morales Morgado. I.S.B.N: 978 84-7800-174-3. Depósito legal: S.1.152-2010.

Morales, E. M., García, F. J. \& Barrón, Á. (2007). Definición pedagógica del nivel de granularidad de Objetos de Aprendizaje. En . López, A.; García, F.; Seoane, A. \& Morales, E. (Eds.). Actas del I Congreso Internacional de Tecnología, Formación y Comunicación (EuniverSALearning’07). (12-14 de septiembre, Salamanca, España).

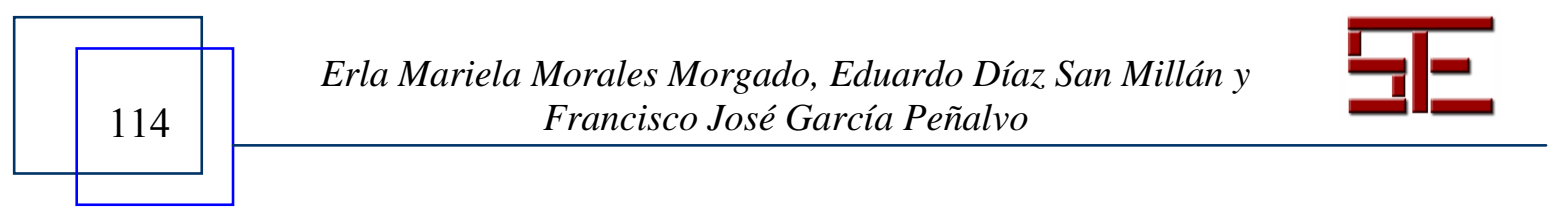




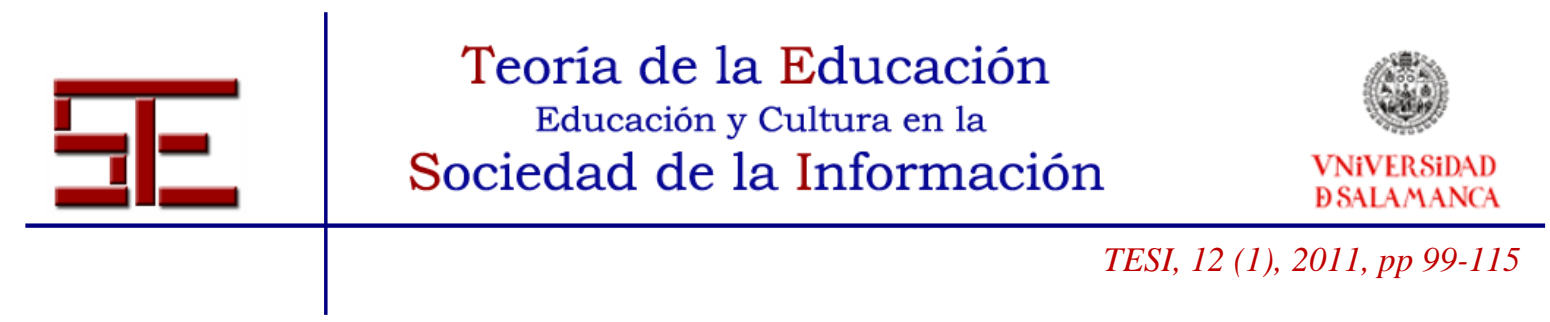

Editorial LOGO. Asociación Española de Estudios sobre Lengua, Pensamiento y Cultura Clásicas. ISBN: 978-84-930218-4-9

Muñoz, J., Ruiz, R.E. \& Álvarez, F. (2007). Evaluación de Objetos de Aprendizaje a través del aseguramiento de Competencias Educativas. Actas del Virtual Educa Brasil 2007. http://e-spacio.uned.es/fez/view.php?pid=bibliuned:19233.

Spencer, L.M. \& Spencer, S. M. (1993). Competence at work. Models for superior performance. New York: John Wiley \& Sons, Inc.

Villa Sánchez, A. \& Poblete Ruiz, M. (2008). Competence-based learning. A proposal for the assessment of generic competences. Universidad de Deusto. Serie Tuning project. ISBN: 978-84-9830-967-6 (formato digital), ISBN: 978-84-9830-198-4 (formato impreso).

Para citar el presente artículo puede utilizar la siguiente referencia:

Morales Morgado E. M., Díaz San Millán E. y García Peñalvo F. J. (2011). Gestión de Objetos de Aprendizaje a través de la Red, basada en el desarrollo de competencias, en Hernández Serrano, M. J. y Fuentes Agustí, M. (Coords.) La red como recurso de información en educación. Revista Teoría de la Educación: Educación y Cultura en la Sociedad de la Información. Vol. 12, $\mathrm{n}^{\circ}$ 1. Universidad de Salamanca, pp. 99-115 [Fecha de consulta: $\mathrm{dd} / \mathrm{mm} / \mathrm{aaaa}]$.

http://campus.usal.es/ revistas_trabajo/index.php/revistatesi/article/view/7825/7852

Erla Mariela Morales Morgado, Eduardo Díaz San Millán y Francisco José García Peñalvo 